\title{
Factores asociados con la presencia de tuberculosis en pacientes con el síndrome de inmunodeficiencia adquirida en Cuba
}

\author{
Andrés Reyes Corcho, ${ }^{1}$ Manuel Díaz Jidy, ${ }^{2}$ Antonio Pérez Rodríguez, ${ }^{2}$ \\ Yadira Bouza Jiménez ${ }^{3}$ y Yanelka Bouza Jiménez ${ }^{1}$
}

Forma de citar Reyes Corcho A, Díaz Jidy M, Pérez Rodríguez A, Bouza Jiménez Y, Bouza Jiménez Y. Factores asociados con la presencia de tuberculosis en pacientes con el síndrome de inmunodeficiencia adquirida en Cuba. Rev Panam Salud Publica. 2004;15(5):341-7.

RESUMEN Objetivos. Determinar los factores asociados con el desarrollo de la tuberculosis en pacientes con el síndrome de inmunodeficiencia adquirida (sida) e identificar los sintomas y signos de tuberculosis más frecuentes en este grupo de pacientes.

Métodos. Se realizó un estudio observacional retrospectivo de casos y testigos en 143 pacientes con diagnóstico de sida egresados del Instituto de Medicina Tropical Pedro Kourí, de Ciudad de La Habana, Cuba, entre enero de 1997 y marzo de 2001. El grupo de casos estuvo constituido por los 72 pacientes con sida que presentaban alguna de las formas clinicas de tuberculosis, mientras que el grupo testigo estuvo integrado por los primeros 71 pacientes que egresaron con sida sin tuberculosis. Se evaluaron las siguientes variables: estado clínico con relación al sida previo al estudio, infecciones oportunistas mayores padecidas antes del diagnóstico de tuberculosis (pneumocistosis pulmonar, toxoplasmosis cerebral, candidiasis profunda, isosporidiosis y neumonía recurrente), concentración de linfocitos T CD4+, y sintomas y signos clínicos de tuberculosis. Los datos primarios se tomaron de los expedientes clínicos de los pacientes. Se calcularon la frecuencia de las variables cualitativas nominales y las razones brutas de posibilidades (OR) y sus intervalos de confianza de 95\%. La asociación estadística entre las variables se determinó mediante la prueba de ji al cuadrado con la corrección de Yates. El efecto individual de cada variable se evaluó mediante análisis de regresión logística con múltiples variables. El nivel de significación estadística fue de 0,05.

Resultados. La tuberculosis en este grupo de pacientes mostró una asociación estadísticamente significativa con la condición de estar enfermo de sida antes del estudio $(O R=3,57$; IC95\%: 1,78 a 7,17); con tener antecedentes de pneumocistosis pulmonar (OR =4,73; IC95\%: 1,51 a 15,76), toxoplasmosis cerebral (OR $=6,22$; IC95\%: 1,21 a 42,99) y candidiasis profunda $(O R=11,29$; IC95\%: 1,40 a 246,5); y con tener concentraciones de linfocitos T CD4+ inferiores a 200 células $/ \mathrm{mm}^{3}$. Sin embargo, la regresión logística solo mostró una asociación significativa con los antecedentes de candidiasis profunda (OR = 10,47; IC95\%: 1,06 a 103,5; $\mathrm{P}=0,0446)$. Los síntomas asociados con el diagnóstico clínico de tuberculosis fueron hemoptisis $(O R=7,54$; IC95\%: 1,88 a 170,34), fiebre de origen desconocido $(O R=13,38 ;$ IC95\%: 5,55 a 32,96), sudación nocturna $(O R=21,95 ;$ IC95\%: 4,66 a 142,43) y pérdida de peso $(O R=$ 3,52; IC95\%: 1,65 a 7,55), mientras que los signos asociados fueron las linfadenopatías regio-

1 Hospital Universitario Clínico Quirúrgico Dr. Gustavo Aldereguía Lima, Cienfuegos, Cuba. La correspondencia debe dirigirse a Andrés Reyes Corcho a la siguiente dirección postal: Calle Lombart No. 9, entre Vila y Cienfuegos, Cumanayagua,
Cienfuegos, Cuba. Correo electrónico: andresrc@ cmy.cfg.sld.cu

2 Instituto de Medicina Tropical Dr. Pedro Kourí, Ciudad de La Habana, Cuba.
3 Hospital Pediátrico Universitario Paquito González Cueto, Cienfuegos, Cuba. 
nales (OR $=10,00 ;$ IC95\%: 1,22 a 220,3), la hepatomegalia (OR = 5,44; IC95\%: 1,76 a 17,95) y la esplenomegalia $(O R=5,08$; IC95\%: 1,63 a 16,83).

Conclusiones. Los sintomas y signos observados con más frecuencia en los pacientes con sida y tuberculosis son los característicos de esta enfermedad en pacientes sin sida. A pesar de que en pacientes con sida la tuberculosis puede encontrarse asociada con otras enfermedades que producen sintomas parecidos, los presentes resultados indican que los sintomas tradicionales de tuberculosis pueden ayudar a diagnosticar la enfermedad en este grupo de enfermos.

Palabras clave
La tuberculosis es una de las enfermedades infecciosas de más amplia distribución en el mundo y constituye una de las primeras causas de muerte en pacientes con el síndrome de inmunodeficiencia adquirida (sida), especialmente en países pobres. Según datos de la Organización Mundial de la Salud (OMS), un tercio de la población mundial está infectada por $\mathrm{Myco-}$ bacterium tuberculosis y cada año se infectan alrededor de 8 millones de personas, de las cuales mueren cerca de 2 millones $(1,2)$. Sin embargo, la situación no es igual en todos los países: mientras que en los Estados Unidos de América se logró reducir la incidencia de tuberculosis en el año 2000 a la cifra más baja que jamás haya tenido ese país (5,8 casos por 100000 habitantes) después del aumento sostenido del número de casos observado hasta el año 1992 (3), la incidencia ha seguido aumentando en los 22 países donde se concentra el $80 \%$ de los casos de tuberculosis en el mundo, entre ellos Bangladesh, Brasil, China, Haití, Rusia y los países de África subsahariana $(4,5)$.

Las mejoras socioeconómicas logradas en muchos países después de la Segunda Guerra Mundial y el descubrimiento de potentes tuberculostáticos a finales de la década de 1940 permitieron reducir la mortalidad y la morbilidad por tuberculosis. Sin embargo, desde la década de 1980, estos avances se han visto menoscabados por la pérdida de prioridad de los programas de control de la enfermedad, la situación de pobreza e insalubridad predominante en muchos países, la carencia de recursos financieros y humanos dedicados a la vigilancia y al control de la tuberculosis y la interacción de esta enfermedad con el sida (5-9). Este con- junto de factores favoreció la reemergencia de la tuberculosis en la última década del siglo pasado.

La propagación de la infección por el virus de la inmunodeficiencia humana (VIH) y del sida ha contribuido a agudizar el impacto de la tuberculosis (10). Se calcula que alrededor de 8 millones de casos de tuberculosis y 2900000 de las defunciones ocasionadas por esta enfermedad entre 1991 y 2000 han estado asociados con la infección por el VIH (11). En la actualidad, alrededor de 13 millones de personas tienen ambas infecciones simultáneamente, de ellas 9,5 millones viven en África subsahariana y 2,3 millones en el sudeste asiático. En estas regiones, donde la prevalencia de la infección por el VIH sobrepasa el 30\%, la infraestructura sanitaria es deficiente y el acceso a tratamientos eficaces contra ambas enfermedades es muy limitado $(11,12)$.

En presencia del sida, las manifestaciones clínicas de la tuberculosis se exacerban y se dificulta el diagnóstico microbiológico por la reducción del número de bacilos presente en las muestras de esputo. Esto se observa especialmente en las formas graves extrapulmonares y diseminadas de la enfermedad, que representan $50 \%$ de todos los casos de tuberculosis notificados en regiones con altas prevalencias de sida y de tuberculosis $(2,13-16)$. Otro fenómeno asociado es la presencia cada vez más frecuente de pacientes de tuberculosis con baciloscopia negativa, quienes suelen tener una peor evolución clínica y un mayor grado de deterioro inmunológico $(17,18)$.

Aunque en Cuba también se ha observado la diseminación de la tuberculosis como enfermedad reemergente, la baja prevalencia de la infección por
VIH en el país $(0,05 \%)$ y la existencia de un programa nacional consolidado para la prevención y el control de la tuberculosis hacen que las implicaciones clínicas y sociales de la coinfección sean menores que en otros países en desarrollo $(19,20)$. Las investigaciones realizadas en Cuba acerca del desarrollo, la evolución y el pronóstico de la tuberculosis en los pacientes infectados por VIH demuestran que la tuberculosis en estas personas presenta una variada expresión clínica, relacionada con grados extremos de depresión inmunológica, generalmente precedida por varias infecciones oportunistas (21-24).

No obstante, aún se desconocen algunos aspectos de la tuberculosis asociada con el sida en el contexto cubano, en particular los factores asociados con la adquisición de la enfermedad en este grupo de pacientes. Los objetivos del presente trabajo fueron determinar qué factores se asocian con el desarrollo de la tuberculosis en pacientes con sida e identificar los síntomas y signos de tuberculosis más frecuentes en este grupo de pacientes.

\section{MATERIALES Y MÉTODOS}

Se realizó un estudio observacional retrospectivo de casos y testigos. En la investigación participaron 143 pacientes con diagnóstico de sida según las categorías clínicas e inmunológicas definidas por los Centros para el Control y la Prevención de Enfermedades (CDC) (25). La muestra se compuso de pacientes que habían egresado del Instituto de Medicina Tropical Pedro Kourí (IPK), de Ciudad de La Habana, Cuba, entre enero de 1997 y marzo de 2001. El grupo de casos estuvo consti- 
tuido por los 72 enfermos con sida que presentaban alguna de las formas clínicas de tuberculosis, mientras que el grupo testigo estuvo integrado por los primeros 71 pacientes egresados con sida sin tuberculosis y con antecedentes de alguna infección oportunista definitoria de sida.

El diagnóstico de tuberculosis se realizó mediante el examen directo del frotis de esputo con la tinción de ZiehlNeelsen, el cultivo microbiológico del esputo en medio de Lowenstein-Jensen o el estudio histológico del material obtenido por biopsia o necropsia. Se incluyó en la muestra a pacientes sin comprobación microbiológica si cumplían los criterios clínicos y radiológicos de la Sociedad Estadounidense del Tórax [American Thoracic Society] y del Programa Nacional de Control de la Tuberculosis de Cuba para el diagnóstico del primer episodio de tuberculosis $(19,26)$. En los pacientes fallecidos se verificó el diagnóstico de tuberculosis en la necropsia mediante la detección de bacilos ácido-alcohol resistentes en el tejido o el cultivo en medio de Lowenstein-Jensen.

Se registró el estado de cada enfermo con relación al sida previo al inicio del estudio. Se consideró enfermo de sida a todo paciente seropositivo al VIH que tenía o había tenido alguna de las infecciones oportunistas propias de ese síndrome o que tenía concentraciones de linfocitos T CD4+ menores de 200 células $/ \mathrm{mm}^{3} \mathrm{o}$ ambas cosas. El diagnóstico de las infecciones oportunistas que definen el sida se realizó según la clasificación de los CDC de 1993 (25). Los pacientes se clasificaron en tres grupos según la concentración de linfocitos T CD4+ determinada mediante citometría de flujo: $\geq 500$ células $/ \mathrm{mm}^{3}$, de 200 a 499 células $/ \mathrm{mm}^{3}$ y $<200$ células $/ \mathrm{mm}^{3}$, de acuerdo con la definición de los CDC de 1993 (25).

Además del estado clínico en relación con el sida previo al estudio, se evaluaron las siguientes variables: infecciones oportunistas mayores padecidas antes del diagnóstico de tuberculosis (pneumocistosis pulmonar, toxoplasmosis cerebral, candidiasis profunda, isosporidiosis y neumonía recurrente), concentración de linfoci- tos T CD4+, y síntomas y signos clínicos de tuberculosis (tos, expectoración, hemoptisis, fiebre, fiebre de origen desconocido, sudación nocturna, pérdida de peso, disnea, hepatomegalia, esplenomegalia y linfadenopatías regionales, independientemente de las cadenas ganglionares afectadas).

Se definió como fiebre de origen desconocido una temperatura corporal de $38,3{ }^{\circ} \mathrm{C}$ o más durante un mínimo de cuatro semanas en un paciente ambulatorio o durante un mínimo de tres días en un paciente hospitalizado, tomando en cuenta dos días para descartar diagnósticos probables (27).

Los datos primarios se tomaron de los expedientes clínicos archivados en el Departamento de Registros Médicos del IPK.

De los pacientes que participaron en el estudio, 75,5\% eran hombres; la edad media de los casos fue de 30 años y de los testigos, de 33 años. No se encontraron diferencias significativas entre los dos grupos en cuanto a la edad y la composición por sexos.

\section{Análisis estadístico}

Se utilizó el paquete estadístico Epi Info (versión 6.04) para calcular la frecuencia de las variables cualitativas nominales y las razones brutas de posibilidades (odds ratios, OR), así como sus intervalos de confianza de $95 \%$ (IC95\%). La asociación estadística entre las diferentes variables se determinó mediante la prueba de ji al cua- drado con la corrección de Yates. Para conocer la asociación entre cada variable y la presencia de tuberculosis se realizó el análisis con múltiples variables con la ayuda del programa SPSS (versión 9.0). El nivel de significación estadística fue de 0,05.

\section{RESULTADOS}

Entre las personas examinadas en este estudio, la tuberculosis mostró una asociación significativa con el estado clínico (presencia de sida) antes del estudio (OR = 3,57; IC95\%: 1,78 a $7,17)$; con tener antecedentes de pneumocistosis pulmonar $(\mathrm{OR}=4,73$; IC95\%: 1,51 a 15,76), toxoplasmosis cerebral (OR = 6,22; IC95\%: 1,21 a 42,99) o candidiasis profunda $(\mathrm{OR}=11,29$; IC95\%: 1,40 a 246,5); y con tener concentraciones de linfocitos T CD4+ inferiores a 200 células $/ \mathrm{mm}^{3}$ (cuadro 1). No se encontró ninguna asociación significativa entre la tuberculosis y los antecedentes de isosporidiosis sintomática $(\mathrm{OR}=2,00$; IC95\%: 0,14 a 58,08) ni de neumonía recurrente $(\mathrm{OR}=0,85$; IC95\%: 0,30 a 2,55).

En cuanto a los síntomas observados en los pacientes con tuberculosis, se encontró una asociación significativa $(P<$ $0,05)$ con la presencia de hemoptisis $(\mathrm{OR}=7,54$; IC95\%: 1,88 a 170,34), fiebre de origen desconocido $(\mathrm{OR}=13,38$; IC95\%: 5,55 a 32,96), sudación nocturna $(\mathrm{OR}=21,95$; IC95\%: 4,66 a 142,43) y ninguna pérdida de peso $(\mathrm{OR}=3,52$; IC95\%: 1,65 a 7,55). Los signos asocia-

CUADRO 1. Factores clínicos asociados con el desarrollo de tuberculosis en enfermos de sida. Ciudad de La Habana, Cuba, 1997-2001

\begin{tabular}{lccr}
\hline \multicolumn{1}{c}{ Variable } & $\begin{array}{c}\text { Razón de } \\
\text { posibilidades }\end{array}$ & $\begin{array}{c}\text { Intervalo de confianza } \\
\text { de } 95 \%\end{array}$ & $P^{\mathrm{a}}$ \\
\hline Estado clínico previo (sida) & 3,57 & $1,78-7,17$ & 0,000519 \\
Pneumocistosis pulmonar & 4,73 & $1,51-15,76$ & 0,004086 \\
Toxoplasmosis cerebral & 6,22 & $1,21-42,99$ & 0,02140 \\
Candidiasis profunda & 11,29 & $1,40-246,5$ & 0,01290 \\
Isosporidiosis & 2,00 & $0,14-58,08$ & 0,99023 \\
Neumonía recurrente & 0,87 & $0,30-2,55$ & 0,97388 \\
Concentración de linfocitos T CD4+ & & & $0,00001^{\mathrm{b}}$ \\
\multicolumn{2}{c}{ C20 células/mm } \\
\end{tabular}

a Nivel de significación de 0,05.

${ }^{b}$ Diferencias calculadas entre casos y testigos según los grupos de CD4+. 
CUADRO 2. Síntomas y signos asociados con la presencia de tuberculosis en enfermos de sida. Ciudad de La Habana, Cuba, 1997-2001

\begin{tabular}{lccr}
\hline \multicolumn{1}{c}{ Síntoma o signo clínico } & $\begin{array}{c}\text { Razón de } \\
\text { posibilidades }\end{array}$ & $\begin{array}{c}\text { Intervalo de confianza } \\
\text { de } 95 \%\end{array}$ & $P^{\mathrm{a}}$ \\
\hline Tos & 0,73 & $0,26-2,04$ & 0,66151 \\
Expectoración & 1,52 & $0,69-3,32$ & 0,33619 \\
Hemoptisis & 7,54 & $1,88-170,34$ & 0,03280 \\
Fiebre de origen desconocido & 13,38 & $5,55-32,96$ & $>0,00001$ \\
Fiebre & 0,06 & $0,02-0,17$ & $>0,00001$ \\
Sudación nocturna & 21,95 & $4,66-142,43$ & $>0,00001$ \\
Pérdida de peso & 3,52 & $1,65-7,55$ & 0,00057 \\
Linfadenopatías regionales & 10,00 & $1,22-220,3$ & 0,00921 \\
Hepatomegalia & 5,44 & $1,76-17,95$ & 0,00113 \\
Esplenomegalia & 5,08 & $1,63-16,83$ & 0,00233 \\
Disnea & 0,07 & $0,01-0,25$ & $>0,00001$ \\
\hline
\end{tabular}

a Nivel de significación de 0,05.

dos significativamente con la tuberculosis en estos pacientes fueron las linfadenopatías regionales $(\mathrm{OR}=10,00$; IC95\%: 1,22 a 220,3$)$, la hepatomegalia $(\mathrm{OR}=5,44$; IC95\%: 1,76 a 17,95$)$ y la esplenomegalia $(\mathrm{OR}=5,08$; IC95\%: 1,63 a 16,83) (cuadro 2).

Los resultados del análisis con múltiples variables indicaron que los pacientes que habían padecido candidiasis profunda tenían una probabilidad 10,47 veces mayor de padecer tuberculosis que los que no habían tenido esa infección $(P<0,0446)$. No se encontró asociación significativa independiente entre la tuberculosis y el resto de las variables, como la presencia de sida previo al estudio neumológico ( $\mathrm{OR}=1,04$;
IC95\%: 0,39 a 2,74), los antecedentes de pneumocistosis pulmonar $(\mathrm{OR}=2,97$; IC95\%: 0,82 a 10,73) y de toxoplasmosis cerebral (OR = 4,35; IC95\%: 0,76 a 24,64), o concentraciones de células T CD4+ inferiores a 200 células $/ \mathrm{mm}^{3}(\mathrm{OR}=1,34$; IC95\%: 0,35 a 5,09) (cuadro 3).

Las variables asociadas con el diagnóstico clínico de la tuberculosis fueron la presencia de hemoptisis $(\mathrm{OR}=12,23$; IC95\%: 1,03 a 143,97), fiebre de origen desconocido (OR = 6,74; IC95\%: 2,67 a $17,01)$, sudación nocturna (OR $=7,78$; IC95\%: 1,48 a 40,71$)$ y pérdida de peso $(\mathrm{OR}=2,85 ; 1,12$ a 7,25$)$ (cuadro 4$)$. La esplenomegalia y hepatomegalia presentaron una alta correlación, por lo que se diseñaron dos modelos de regresión

CUADRO 3. Antecedentes asociados con el desarrollo de la tuberculosis en enfermos de sida según, los resultados del análisis de múltiples variables. Ciudad de La Habana, Cuba, 1997-2001

\begin{tabular}{lccc}
\hline \multicolumn{1}{c}{ Variable } & $\begin{array}{c}\text { Razón de } \\
\text { posibilidades }^{\mathrm{a}}\end{array}$ & $\begin{array}{c}\text { Intervalo de confianza } \\
\text { de } 95 \%\end{array}$ & $P^{\mathrm{b}}$ \\
\hline Estado clínico previo (sida) & 1,04 & $0,39,2,74$ & 0,9260 \\
Pneumocistosis pulmonar & 2,97 & $0,82,10,73$ & 0,0959 \\
$\begin{array}{l}\text { Toxoplasmosis cerebral } \\
\text { Candidiasis profunda }\end{array}$ & 4,35 & $0,76,24,64$ & 0,0962 \\
$\begin{array}{l}\text { Concentraciones de linfocitos T CD4+ } \\
\quad \text { de 200 a 499 células/mm }\end{array}$ & 10,47 & $1,06,103,5$ & 0,0446 \\
$\begin{array}{l}\text { Concentración de linfocitos T CD4+ } \\
\quad<200 \text { células/mm }\end{array}$ & 0,34 & $0,08,1,43$ & 0,1439 \\
\hline
\end{tabular}

\footnotetext{
a Modelo de regresión logística.
${ }^{\mathrm{b}}$ Nivel de significación de 0,05 .
}

logística de los que se excluyeron estas variables de manera alternada; en ambos casos se confirmó la asociación estadística de las variables (hemoptisis, fiebre de origen desconocido, sudación nocturna y pérdida de peso) con el diagnóstico clínico de tuberculosis.

\section{DISCUSIÓN}

En el presente estudio se evaluó la asociación entre algunas variables clínicas y el diagnóstico de la tuberculosis en los pacientes con sida.

Los pacientes del grupo testigo presentaban enfermedades oportunistas mayores en el momento del estudio neumológico, por lo que se clasificaron como enfermos de sida. Esto favoreció la homogeneidad entre los casos y los testigos, de manera que la diferencia entre estos dos grupos se basó en la presencia o la ausencia de tuberculosis. Las enfermedades oportunistas más frecuentes fueron, entre otras, pneumocistosis pulmonar, criptococosis diseminada, neumonía recurrente y otras micosis sistémicas.

En el presente estudio se encontró un mayor número de casos de tuberculosis entre los pacientes con sida que tenían antecedentes de pneumocistosis pulmonar, neurotoxoplasmosis o candidiasis profunda o que presentaban concentraciones bajas de linfocitos T CD4+ $\left(<200\right.$ células $\left./ \mathrm{mm}^{3}\right)$, que entre los que no cumplían estas condiciones. Sin embargo, según los resultados de la regresión logística, solo la asociación con los antecedentes de candidiasis profunda fue significativa $(\mathrm{OR}=10,47$; IC95\%: 1,06 a 103,$5 ; P=0,0446$ ). Muchas investigaciones basadas en diseños descriptivos han tratado de encontrar una asociación entre la infección avanzada por VIH y una mayor probabilidad de contraer tuberculosis, pero esta asociación resultó significativa solo en dos estudios controlados: uno de casos y testigos (28) y otro de cohorte (29). En otro estudio, El-Sohl y colaboradores encontraron que tener concentraciones de linfocitos T CD4 $+<200$ células $/ \mathrm{mm}^{3}$ era un fuerte factor pronóstico de tuberculosis activa en enfermos de sida (30). En Minas Gerais, Brasil, se realizó 
CUADRO 4. Signos y síntomas asociados con el diagnóstico clínico de tuberculosis en enfermos de sida, según los resultados del análisis de múltiples variables. Ciudad de La Habana, Cuba, 1997-2001

\begin{tabular}{lccr}
\hline \multicolumn{1}{c}{ Síntoma o signo clínico } & $\begin{array}{c}\text { Razón de } \\
\text { posibilidades }^{\mathrm{a}}\end{array}$ & $\begin{array}{c}\text { Intervalo de confianza } \\
\text { de 95\% }\end{array}$ & $P^{\mathrm{b}}$ \\
\hline Hemoptisis & 12,23 & $1,03,143,97$ & 0,0465 \\
Fiebre de origen desconocido & 6,74 & $2,67,17,01$ & 0,001 \\
Sudación nocturna & 7,78 & $1,48,40,71$ & 0,0150 \\
Pérdida de peso & 2,85 & $1,12,7,25$ & 0,0278 \\
Linfadenopatía regional & 10,06 & $0,83,120,63$ & 0,0685 \\
Esplenomegalia & 1,87 & $0,47,7,42$ & 0,3731 \\
Hepatomegalia & 2,05 & $0,53,7,93$ & 0,2970 \\
\hline
\end{tabular}

a Modelo de regresión logística.

${ }^{\mathrm{b}}$ Nivel de significación de 0,05.

un estudio de casos y testigos con diseño retrospectivo, similar al presentado en este trabajo, en el que se evaluó la asociación de la tuberculosis con la presencia del complejo sintomático relacionado con el sida, antecedentes de neumonía y el número de hospitalizaciones anteriores, pero solo se encontró una asociación independiente entre la tuberculosis y el complejo relacionado con el sida (31). En ese estudio, las concentraciones medias de linfocitos T CD4+ tampoco mostraron una asociación significativa con la presencia de tuberculosis.

Aunque no se demostró una asociación entre la inmunodepresión grave y la presencia de tuberculosis, la concentración de linfocitos T CD4+ siempre se ha utilizado para establecer las pautas del diagnóstico y la profilaxis de las infecciones oportunistas en pacientes con sida. En la práctica clínica continúa siendo importante evaluar al paciente de un modo integral, y las concentraciones de linfocitos T CD4+ pueden aportar información importante para tomar decisiones médicas correctas en el tratamiento de estos pacientes. Estudios prospectivos de cohortes en los que se pueda observar cómo evoluciona esta variable a lo largo del tiempo podrían ayudar a conocer con mayor exactitud en qué medida la depresión inmunitaria de los pacientes con sida y tuberculosis es la causa o el efecto de algunas de estas enfermedades o si es el resultado de la interacción de ambos procesos.

A pesar de la baja prevalencia de la infección por VIH en Cuba y de la existencia del Programa Nacional de Control de la Tuberculosis ya consolidado, en este estudio no se encontraron diferencias con respecto a lo observado en otros países en desarrollo con limitados recursos sociales y sanitarios. De hecho, la tuberculosis en pacientes cubanos con sida se ha visto asociada con el padecimiento simultáneo de varias enfermedades y con la inmunodepresión avanzada de los pacientes.
Los síntomas y signos observados con más frecuencia en los pacientes con sida y tuberculosis (hemoptisis, fiebre de origen desconocido, sudación nocturna y pérdida de peso) indican que los enfermos de esta serie tienen los síntomas y signos característicos de esta enfermedad en pacientes sin sida $(3,32-35)$. A pesar de que en pacientes con sida la tuberculosis puede encontrarse asociada con otras enfermedades cuyos síntomas son parecidos a los de la tuberculosis, haciendo que los mismos pierdan especificidad (29), los presentes resultados indican que los síntomas tradicionales de esta enfermedad pueden ayudar a diagnosticar la tuberculosis en este grupo de enfermos (29, 33, 35).

Es importante señalar que este estudio se realizó antes de que en Cuba se generalizara el uso de la terapia antirretroviral de alta eficacia, lo cual podría haber impedido que los enfermos contrajeran la tuberculosis en un contexto clínico e inmunológico más favorable. El carácter retrospectivo de este estudio y el hecho de que los datos primarios se tomaron de las fichas clínicas de los pacientes podrían haber afectado a la exactitud de los síntomas y signos de tuberculosis y menoscabado su validez, especialmente por tratarse de un grupo de pacientes con síntomas muy diversos debido al sida. No obstante, la existencia de un sólido programa de control de la tuberculosis y la pesquisa sistemática de esta enfermedad han permitido que los enfermos se diagnostiquen tempranamente y que las formas atípicas y diseminadas de esta enfermedad asociada al sida sean menos frecuentes de lo notificado en la literatura internacional.

\section{REFERENCIAS}

1. Dye C, Scheele S, Dolin P, Pathania V, Raviglione M. Consensus statement: global burden of tuberculosis. Estimated incidence, prevalence, and mortality by country: $\mathrm{WHO}$ GLOBAL Surveillance and Monitoring Project. JAMA. 1999;282:677-86.
2. Small PM, Fujiwara PI. Medical progress: management of tuberculosis in the United States. N Engl J Med. 2001;345(3):189-200.

3. Division of Tuberculosis Elimination. Surveillance reports: reported tuberculosis in the United States, 2000. Atlanta: Centers for Dis- ease Control and Prevention; 2001. Hallado en: http://www.cdc.gov/nchstp/tb/surv/ surv2000/default.htm. Acceso el 14 de abril de 2004.

4. Santoro-Lopez G, Felix de Pinho AM, Harrison LH, Schechter M. Reduced risk of tuber- 
culosis among Brazilian patients with advanced human immunodeficiency virus infection treated with highly active antiretroviral therapy. Clin Infect Dis. 2002;34:543-6.

5. Farmer P. The major disease in the world to treat or not to treat? N Engl J Med. 2001; 345(3):208-10.

6. Armas Pérez L, González Ochoa E. Manejo de la tuberculosis en la República de Cuba. Rev Cubana Med Trop. 1998;50(2):150-8.

7. Van Cleeff MR, Chum HJ. The proportion of tuberculosis cases in Tanzania attributable to human immunodeficiency virus. Int J Epidemiol. 1995;24:637-42.

8. Geiter L. Ending neglect: the elimination of tuberculosis in the United States. Washington, D.C.: National Academy Press; 2000.

9. De Cock KM, Binkin NJ, Zuber PL, Tappero JW, Castro KG. Research issues involving HIV-associated tuberculosis in resource-poor countries. JAMA. 1996;276:1502-7.

10. Felix de Pinho AM, Santoro-Lopez G, Harrison LH, Schechter M. Chemoprophylaxis for tuberculosis and survival of HIV infected patients in Brazil. AIDS. 2001;15:2129-35.

11. Bentwich Z, Maartens G, Torten D, Lal AA, Lal RB. Concurrent infections and HIV pathogenesis. AIDS. 2000;14:2071-81.

12. Essex M. Human immunodeficiency viruses in the developing world. Adv Virus Res. 1999; 53:71-88.

13. Leonard MK, Larsen N, Drechsler H, Blumberg H, Lennox JL, Arrellano F, et al. Increased survival of persons with tuberculosis and human immunodeficiency virus infection, 1991-2000. Clin Infect Dis. 2002;43: 1002-7.

14. Collins KR, Quinones-Mateu ME, Toossi Z, Arts EJ. Impact of tuberculosis on HIV-1 replication, diversity, and disease progression. AIDS Rev. 2002;4(3):165-76.

15. Dean GL, Edwards SG, Ives NJ, Matthews G, Fox EF, Navaratne L, et al. Treatment of tuberculosis in HIV-infected persons in the era of highly active antiretroviral therapy. AIDS. 2002;16:75-83.

16. Garcia de Olalla P, Martinez-Gonzalez MA, Cayla JA, Jansa JM, Iglesias B, Guerreo R, et al. Influence of highly active anti-retroviral therapy (HAART) on the natural history of extrapulmonary tuberculosis in HIV patients. Int J Tuberc Lung Dis. 2002;6(12):1051-7.
17. Hargreaves NJ, Kadzakumanja O, Whitty CJM, Salaniponi FML, Harries AD, Squire SB. Smear-negative pulmonary tuberculosis in DOTS programme: poor outcomes in an area of high HIV seroprevalence. Int J Tuberc Lung Dis. 2001;5(9):847-54.

18. Colebunders R, Bastian I. A review of the diagnosis and treatment of smear-negative pulmonary tuberculosis. Int J Tuberc Lung Dis. 2000;4(2):97-107.

19. Dotres Martínez C, Pérez González R, Santín Peña M, Marrero Figueroa A, eds. Programa Nacional de Control de la Tuberculosis. La Habana: Editorial Ciencias Médicas; 1999

20. Díaz Jidy M, González Núñez I, Saladrigas Socarrás C, Pérez Ávila J, Millán Marcelo JC, Valdivia Álvarez JA. Coinfección HIV/TB en Cuba. Rev Cubana Med Trop. 1996;48(3): 214-7.

21. Torres R, Fiol J, Carreras L, Pérez J, Hernán$\operatorname{dez} \mathrm{O}$, Marrero A, Gil A, et al. La infección por el virus de la inmunodeficiencia humana y la tuberculosis en Cuba. Bol Oficina Sanit Panam. 1995;119(1):66-73.

22. Brauburger S. Clinical response to tuberculosis therapy in human immunodeficiency virus disease [tesis de maestría en Infectología y Enfermedades Tropicales]. La Habana: Instituto de Medicina Tropical Pedro Kourí; 1998.

23. Alfonso Hernández B. Comportamiento de la tuberculosis en los pacientes infectados por el virus de la inmunodeficiencia humana [tesis de maestría en Infectología y Enfermedades Tropicales]. La Habana: Instituto de Medicina Tropical Pedro Kourí; 1996.

24. Rojas Gómez LJ. Asociación entre la tuberculosis y el virus de la inmunodeficiencia humana en Cuba. Estudio clínico epidemiológico [tesis de maestría en Infectología y Enfermedades Tropicales]. La Habana: Instituto de Medicina Tropical Pedro Kourí; 1997.

25. Costa Pérez Herrero JR, Ocaña Rivera I. Síndrome de inmunodeficiencia adquirida del adulto. En: Rodés Teixidor J, Guardia Massó J, eds. Medicina Interna. 1. ․ㅡ ed. Barcelona: Masson; 1997. Pp. 1689-97.

26. Fitzgerald DW, Desvarieux M, Severe $P$, Joseph P, Johnson WD, Pape JW. Effect of post-treatment isoniazid on prevention of recurrent tuberculosis in HIV-1 infected individuals: a randomised trial. Lancet. 2000;356: $1488-9$.
27. Durack DT, Street AC. Fever of unknown origin reexamined and redefined. En: Remington JS, Swartz MN, eds. Current clinical topics in infections disease. Boston: Blackwell; 1991. Pp. 46-59.

28. Soriano E, Mallolas J, Gatell JM, Latorre X, Miró JM, Peccchiar M, et al. Characteristics of tuberculosis in HIV-infected patients. A case control study. AIDS. 1998;2:429-32.

29. Martin G, Lazarus A. Epidemiology and diagnosis of tuberculosis: recognition of at-risk patients is key to prompt detection. Postgrad Med. 2000;108(2):42-54.

30. El-Sohl A, Mylotte J, Sherif S, Serghani J, Grant BJ. Validity of a decision tree for predicting active pulmonary tuberculosis. Am J Respir Crit Care Med. 1997;155:1711-6.

31. De Castro Toledo AC, Greco DB, Antunes CM. Risk factors for tuberculosis among human immunodeficiency virus-infected persons. A case-control study in Belo Horizonte, Minas Gerais, Brazil (1985-1996). Mem Inst Oswaldo Cruz. 2000;95(4):437-43.

32. Raviglioni MC, $\mathrm{O}^{\prime}$ Brien RJ. Tuberculosis. En: Fauci AS, Braunwald E, Isselbacher KJ, Wilson JD, Martin JB, Kasper DL, et al., eds. Harrison. Principios de Medicina Interna. 14. ${ }^{\mathrm{a}}$ ed. Madrid: Mc Graw Hill-Interamericana; 1998. Pp. 1149-61.

33. Jerant AF, Bannon M, Rittenhouse S. Identification and management of tuberculosis. Am Fam Physician. 2000;61:2667-82.

34. Baraia-Etxaburu J, Santamaría Jáuregui JM. Enfermedades causadas por micobacterias. En: Álvarez-Mon Soto M, Vera López E, Cano Ballesteros JC, eds. Enfermedades infecciosas. Madrid: IDEPSA; 1998. Pp. 399-404.

35. American Thoracic Society, Centers for Disease Control and Prevention. Diagnostic standards and classification of tuberculosis in adults and children. Am J Respir Crit Care Med. 2000;161:1376-95.

Manuscrito recibido el 27 de octubre de 2003. Aceptado para publicación, tras revisión, el 1 de marzo de 2004. 
ABSTRACT Objectives. To determine the factors associated with the development of tuberculosis in patients with acquired immunodeficiency syndrome (AIDS) and to identify the most frequent symptoms and signs of tuberculosis in this group of patients.

\section{Factors associated with the presence of tuberculosis in patients with acquired immunodeficiency syndrome in Cuba}

Methods. This retrospective observational case-control study was carried out with 143 patients diagnosed with AIDS who were discharged from the Pedro Kourí Institute of Tropical Medicine, which is in the city of Havana, Cuba, between January 1997 and March 2001. The cases were 72 patients with AIDS and some clinical form of tuberculosis, while the control group was made up of the first 71 AIDS patients without tuberculosis who were discharged. The following variables were evaluated: AIDS stage before the study, serious opportunistic infections suffered before the diagnosis of tuberculosis (pulmonary pneumocystosis, cerebral toxoplasmosis, systemic candidiasis, isosporiasis, and recurrent pneumonia), concentration of CD4+ T lymphocytes, and clinical symptoms and signs of tuberculosis. The primary data were taken from the clinical files of the patients. We calculated the frequency of the nominal qualitative variables and the crude odd ratios (ORs) and their 95\% confidence intervals (CIs). The statistical association among the variables was determined with the chisquare test with Yates correction. The individual effect of each variable was assessed through multivariate logistic regression analysis. The level of statistical significance was 0.05 .

Results. Tuberculosis in this group of patients showed a statistically significant association with: being ill with AIDS before the study (OR $=3.57 ; 95 \%$ CI: 1.78 to 7.17 ); a history of pulmonary pneumocystosis (OR $=4.73$; 95\% CI: 1.51 to 15.76), cerebral toxoplasmosis (OR $=6.22 ; 95 \% \mathrm{CI}: 1.21$ to 42.99$)$, or systemic candidiasis $(\mathrm{OR}=11.29$; 95\% CI: 1.40 to 246.5); and having CD4+ T lymphocyte concentrations lower than 200 cells $/ \mathrm{mm}^{3}$. However, the logistic regression showed a significant association only with the history of systemic candidiasis ( $\mathrm{OR}=10.47 ; 95 \% \mathrm{CI}$ : 1.06 to $103.5 ; P=0.0446)$. The symptoms associated with the clinical diagnosis of tuberculosis were hemoptysis $(\mathrm{OR}=7.54 ; 95 \% \mathrm{CI}: 1.88$ to 170.34$)$, fever of unknown origin $(\mathrm{OR}=13.38 ; 95 \% \mathrm{CI}: 5.55$ to 32.96$)$, night sweats $(\mathrm{OR}=21.95 ; 95 \% \mathrm{CI}: 4.66$ to 142.43$)$, and weight loss $(\mathrm{OR}=3.52$; 95\% CI: 1.65 to 7.55$)$. The associated signs were regional lymphadenopathies (OR = 10.00; $95 \%$ CI: 1.22 to 220.3 ), hepatomegaly (OR $=5.44 ; 95 \% \mathrm{CI}: 1.76$ to 17.95 ), and splenomegaly (OR $=5.08 ; 95 \%$ CI: 1.63 to 16.83$)$.

Conclusions. The symptoms and signs seen most frequently in the patients with AIDS and tuberculosis are the characteristics of tuberculosis in patients without AIDS. In patients with AIDS, tuberculosis can be associated with other diseases whose symptoms are similar to those of tuberculosis. Nevertheless, these results indicate that the traditional symptoms of tuberculosis can help diagnose tuberculosis in this group of patients.

\begin{tabular}{|c|c|}
\hline 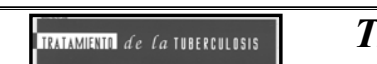 & atamiento de la Tuberculosis. Directrices para los programas nacionales, 2a ed \\
\hline 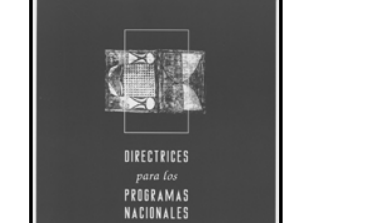 & $\begin{array}{l}\text { En esta obra se facilitan normas prácticas formuladas por expertos para el tratamiento y } \\
\text { control de la enfermedad en el contexto de los programas antituberculosos nacionales. } \\
\text { Esta segunda edición del manual ha sido revisada para reflejar la gran experiencia } \\
\text { adquirida, desde } 1993 \text {, con el uso de las estrategias de control recomendadas por la } \\
\text { Organización Mundial de la Salud. }\end{array}$ \\
\hline (む) & El manual ofrece a los directores de programas, a los encargados de formular las \\
\hline $\begin{array}{l}\text { 1997, 79p., } \\
\text { Código: WHO } 17 \\
\text { Precio: US\$11.00 / US\$ } 8.00 \\
\text { en América Latina y el Caribe }\end{array}$ & $\begin{array}{l}\text { para el control de la tuberculosis que depende de definiciones de caso precisas, cate- } \\
\text { gorías terapéuticas bien definidas y regímenes de tratamiento estandarizados a base de } \\
\text { medicamentos antituberculosos esenciales. }\end{array}$ \\
\hline & \\
\hline
\end{tabular}

